\title{
Citations
}

\section{Proof of Absence}

Defining the interactome in a credible, efficient way remains a daunting task. In silico methods are unlikely to pick up every biological interaction, particularly if the binding event is preceded by a conformational change. In vitro interaction assays, meanwhile, rely on the slow and often laborious production of purified protein. Although the yeast two-hybrid system overcomes some of these disadvantages, it is itself limited in that it can provide relatively little information about interaction surfaces. Writing in the Journal of Molecular Biology, Dhayalan et al. describe how a new approach they call "absence of interference" can strengthen the informational content provided by yeast two-hybrid methods. The strategy takes one member of a pair of interacting proteins and subjects it to random mutagenesis. The resulting mutants are then tested in the yeast two-hybrid assay, and all those mutant proteins that do not disrupt the interaction are analyzed. This creates a "negative footprint," as mutations in the interaction region would be expected to be absent from

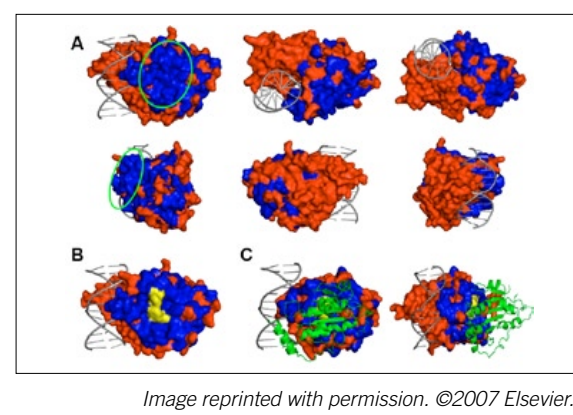

the list of binding-competent mutants. Assuming that structural information is available for the mutagenized protein, the probable interaction domain can be visualized. Dhayalan et al. test their system by studying the interaction between the DNA methyltransferase Dnmt3a and its binding partner Dnmt3L, a regulator of the enzyme. Following error-prone PCR, a library of Dnmt3a mutants was created and tested for interaction with Dnmt3L by yeast two-hybrid. As expected, most library members were no longer able to support the interaction. Those with preserved binding were reanalyzed to remove false positives, and a total of 76 clones were sequenced, showing 648 mutations. A web-based package for displaying the results was developed and used to map these mutations onto the surface of an existing Dnmt3L structural model. This process revealed a 500-600 $\AA^{2}$ region in which no mutations were observed; as expected, this area is topologically separate from the binding sites for cofactor and DNA. Most impressively, the interaction site prediction was confirmed by the fortuitous publication of independent work describing the structure of the interacting domains of these two proteins as determined by $X$-ray crystallography. This simple yet powerful strategy should be appealing to those who have uncovered interacting proteins by yeast two-hybrid. For those with existing mutagenesis data who wish to take advantage of the mapping tool, the protein interface mapper is available at biochem. jacobs-university.de/prima/.

\section{Dhayalan et al. Mapping of protein-protein interaction sites by the 'Absence of Interference' approach. Journal of Molecular Biology [Epub ahead of print, December 17, 2007; doi:10.1016/j.jmb.2007.12.032].}

\section{Think Global and Local}

Active membrane transport proteins are thought to function by the concerted action of global conformational changes in the protein and local alterations in the substrate binding site. It is believed that the overall shape of the membrane protein initially favors the substrate binding within a site accessible at one side of the membrane, and after an overall conformational change, opens up the substrate binding site to the other side of the membrane. However attractive this model is, there are no existing methods that allow structural probing of global protein structure in conjunction with detailed analysis of the alterations within the substrate binding site. In a recent publication in Structure, Andersson et al. propose that time-resolved X-ray scattering methods may be useful in revealing both global and local conformational changes. Although low-angle scattering data provide insights on the more global structural changes, strategic placement of heavy atoms will generate regions of strongly scattered X-rays that will help indicate the localized movements within that vicinity. To model their hypothesis, the authors selected halorhodopsin, which functions in light- driven halide transport. Although this membrane protein normally functions to import chloride ions into the cell, it can also transport iodide ions. This is significant, because iodide can serve as a heavy atom site, providing more detailed information about the movements involving the substrate site. Molecular dynamics simulations and X-ray scattering values were performed, revealing that the use of two heavy atoms would be sufficient to detect local structural changes with medium-scattering angles. In addition, low-scattering angles should be able to detect helical movements consistent with more global changes. This proposed strategy is expected to be generalizable to other systems in which heavy atom labeling is possible. In light of intense interest in membrane transport proteins as potential drug targets, the ability to see the forest and the trees in terms of protein structure should be enormously influential.

Andersson et al. 2008. A proposed time-resolved X-ray scattering approach to track local and global conformational changes in membrane transport proteins. Structure 16:21-28. 


\section{Citations}

\section{Accelerating the PIPEline}

Shortening the time needed for structural genomics initiatives will require better methods for truncating proteins. Although it might seem undesirable to determine the structure of a prematurely truncated protein, full-length targets may not crystallize well. Even so, it is often difficult to predict exactly where to terminate the protein in order to make a crystallizable product. For that reason, producing many truncated variants and then screening them for crystallization success is an attractive approach. In an article appearing in Proteins: Structure, Function, and Bioinformatics, Klock et al. describe a comprehensive strategy for producing and screening truncated proteins for use in an X-ray crystallography initiative. Their pipeline begins with PIPE, or polymerase incomplete primer extension. This cloning method is based on the fact that primer extension is not always complete during PCR amplification. As a result, in a subset of molecules, the $5^{\prime}$ sequence of the primer will remain single-stranded and open to annealing with a complementary sequence. If a viable plasmid can be pieced together from PCR products with suitably designed primer pairs, bacterial repair mechanisms will complete the cloning or mutagenesis process posttransformation. In this way, it is possible to rapidly gener- ate a number of protein variants that can be tested for suitability for structural studies. It is this screening process that forms the second element of the proposed structural genomics workflow. Expressed proteins were subjected to liquid chromatography mass spectrometry (LC-MS) to verify the identity of the protein and were scored by an analytical size-exclusion chromatography method. These steps helped define a set of truncation mutants that would be most likely to yield crystal structures. Ninety-six targets were selected for truncation studies; for each target, $12 \mathrm{~N}$-terminal and $12 \mathrm{C}$-terminal truncations were attempted, of which $93 \%$ were successful as tested by picking just one colony. A total of 18 targets yielded structures (13 based on full-length protein), reflecting an overall success rate of $32 \%$.

These results, combined with the speed with which the proteins were prepared, suggests that PIPE cloning and analytical size-exclusion chromatography should speed the push from gene to structure.

Klock et al. Combining the polymerase incomplete primer extension method for cloning and mutagenesis with microscreening to accelerate structural genomics efforts. Proteins: Structure, Function, and Bioinformatics [Epub ahead of print, November 14, 2007; doi: 10.1002/prot.21786].

\section{Dishworthy}

Establishing an efficient and broadly applicable prion replication assay in tissue culture dishes instead of indicator animals is big news. Prion proteins - which are thought to be the infectious agent behind transmissible spongiform encephalopathies like new-variant Creutzfeldt-Jakob disease-are frequently titred in animals. However, this is a time-consuming and expensive system, as visible indications of infection may take a long time to appear. An in vitro alternative is the so-called scrapie cell assay in endpoint format; however, this system cannot support the replication of all prion strains, most likely because of the lack of multiple cell types in the culture dish. In a paper appearing in Nature Neuroscience, Falsig et al. split the difference between in vivo and in vitro replication systems, describing an ex vivo approach that relies upon long-term culture of

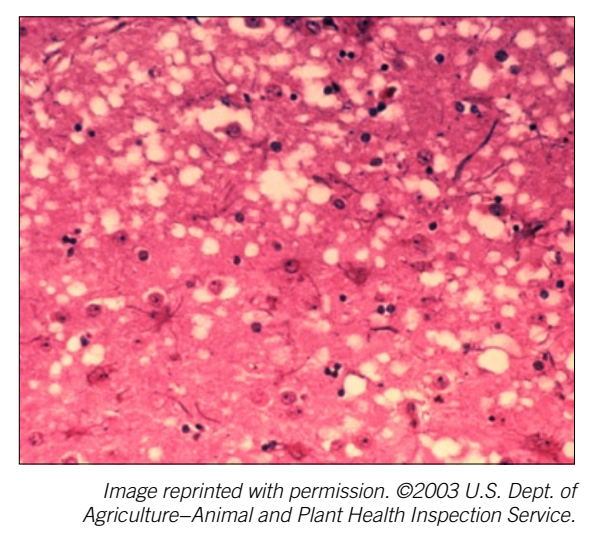

mouse cerebellar slices. Brains from 9- to 12-day-old mouse pups were embedded in agarose, cut with a vibratome, and placed in 24-well plates. These cultures were exposed to brain homogenates from infected or uninfected animals. After 5 weeks of culture, Western blot analysis was used to detect replicated prion protein. Although the new assay proved to be about an order of magnitude less sensitive than the previously described in vitro system, the ex vivo method could be made equally sensitive if microglia were depleted. This could be achieved by preparing brain slices from a mouse strain in which microglia can be ablated by treatment with ganciclovir. Moreover, the ex vivo system allowed replication of a wider variety of transmissible spongiform encephalopathy strains than the in vitro assay. Advantages over the in vivo system include a 5 -fold faster readout and the ability to perform side-by-side replication assays using brain material from the same animal. This advance should significantly help facilitate research on this serious and perplexing disease agent.

Falsig et al. 2008. A versatile prion replication assay in organotypic brain slices. Nature Neuroscience 11:109-117.

- Selected and written by Nijsje Dorman, a freelance writer in Boston, MA. 